\title{
Endoscopic-Assisted Canal Wall-up Tympanomastoidectomy for Reduction of Residual Cholesteatoma
}

\author{
Mauricio Noschang Lopes Silva1® ${ }^{10}$ Fábio André Selaimen ${ }^{10}$ Felipe da Costa Huve ${ }^{10}$ \\ Fernanda Dias Toshiaki Koga ${ }^{1}$ Luciana Lima Martins-Costa ${ }^{1}$ (1) João Augusto Polesi Bergamaschi ${ }^{1}$ \\ Alice Lang Silva1이 Sady Selaimen da Costa ${ }^{20}$
}

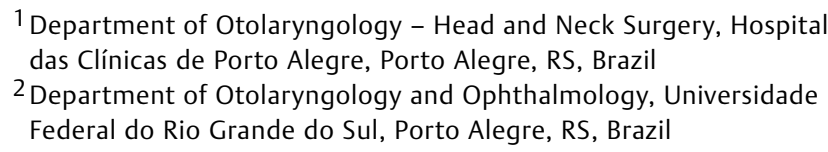

Int Arch Otorhinolaryngol 2022;26(2):e260-e264.

Address for correspondence Mauricio Noschang Lopes Silva, PhD, Hospital de Clínicas de Porto Alegre, Porto Alegre, RS, Brazil (e-mail: noschangmauricio@gmail.com).

\begin{abstract}
Introduction The treatment of cholesteatoma is generally surgical, and the major obstacle is the high prevalence of recidivism. The endoscopic ear surgery technique is proposed to minimize this problem.

Objectives To utilize endoscopes to visualize and manipulate cholesteatoma residues after microscopic removal

Methods Cross-sectional study. Thirty-two patients with cholesteatoma underwent microscopic wall-up mastoidectomy combined with the endoscopic approach. The subjects were assessed for the presence and location of covert disease.

Results Of the 32 cases, 17 (53.12\%) had residual cholesteatoma in the endoscopic phase. Minimal disease was found, usually fragments of the cholesteatoma matrix. Pars

Keywords

- endoscopic ear surgery

- cholesteatoma

- level of evidence: 3 tensa cholesteatomas had more covert disease than pars flaccida cholesteatomas (62.50\% vs $43.75 \%)$. Posterior recesses $(47.05 \%)$ and tegmen tympani $(41.17 \%)$ were the locations with more covert disease $(p<0.05)$.

Conclusion Cholesteatomas of the pars tensa presented more residual disease and were significantly more common in the posterior recesses and tegmen tympani.
\end{abstract}

\section{Introduction}

Schuknecht, in 1974, defined cholesteatoma as the presence of exfoliated keratin in any pneumatized portion of the temporal bone. Ferlito and colleagues (1997) characterized it as an epidermoid cyst, with an independent and progressive growth, involving a destruction of adjacent tissues, especially bone. ${ }^{1}$ Cholesteatomas generally follow growth patterns that are related to the structures of their place of origin and the routes they will proceed. Understanding these growth patterns is very important because cholesteatomas occupy different areas, destroy different structures, and, therefore, present different prognoses. The main routes described by Jackler, ${ }^{2}$ and later modified by Rosito, ${ }^{3}$ are as follows:
1. Posterior epitympanic
2. Posterior mesotympanic

received

October 14, 2020

accepted

March 3, 2021

published online

October 19, 2021
DOI https://doi.org/ 10.1055/s-0041-1730455. ISSN 1809-9777.

\footnotetext{
(c) 2021. Fundação Otorrinolaringologia. All rights reserved. This is an open access article published by Thieme under the terms of the Creative Commons Attribution-NonDerivative-NonCommercial-License, permitting copying and reproduction so long as the original work is given appropriate credit. Contents may not be used for commercial purposes, or adapted, remixed, transformed or built upon. (https://creativecommons.org/ licenses/by-nc-nd/4.0/) Thieme Revinter Publicações Ltda., Rua do Matoso 170, Rio de Janeiro, RJ, CEP 20270-135, Brazil
} 


\section{Anterior epitympanic}

4. Two-route cholesteatoma

5. Undetermined cholesteatomas

The treatment of cholesteatoma is generally surgical, and the major obstacle is the high prevalence of recidivism. Therefore, choosing a conservative surgical technique that yields reestablishment of hearing and provides curative treatment is warranted. The dissemination of endoscopic ear surgery techniques is proposed to collaborate in this purpose. The main rationale is better visualization of the structures as compared to using the microscope alone. ${ }^{4}$ As the microscope only provides a linear view, with the beam of light perpendicular to the structures, the deeper, angled recesses cannot be reached adequately. Essentially, endoscopes comprise several angulations and allow visualization of any region of the middle ear. This advantage could be useful for identifying disease entities that may become recurrences in the future. The aim of the present study was to utilize endoscopes to visualize and manipulate cholesteatoma residues after microscopic removal, to describe the sites of covert disease, and to evaluate the relationships of the different growth patterns.

\section{Methods}

This is a cross-sectional study design. Thirty-two patients diagnosed with cholesteatoma, of ages ranging between 8 and 73 years, underwent surgery in a tertiary hospital in Southern Brazil. The inclusion criteria included cholesteatomas with an indication for canal wall-up (CWU) tympanomastoidectomy. The indications for CWU tympanomastoidectomy comprised the absence of a sclerotic mastoid and gross bone erosions or complications in the preoperative evaluation. Patients with malformations of the temporal bone, other pathologies in the middle ear, and previous ear surgery were excluded from the study. The eligible patients underwent surgery using the conventional CWU tympanomastoidectomy using a microscope. After performing a posterior tympanotomy and removing any visible disease with a microscope, the endoscopic phase began. Karl Storz $4 \mathrm{~mm}$ and $18 \mathrm{~cm}$ endoscopes (Karl Storz SE \& Co KG, Tuttlingen, Germany) at angles of $0^{\circ}$ and $30^{\circ}$, respectively, were used.

Endoscopic-assisted CWU tympanomastoidectomy technique:

1. Retroauricular incision

2. Drilling of the mastoid and opening of the facial recess in all patients

3. Preservation of the posterior wall of the external auditory canal (EAC)

4. Removal of any visible cholesteatoma. A routine thorough review of all middle ear compartments is performed, as recommended by Costa et al. ${ }^{5}$ Careful observation of the main structures of the middle ear, such as the protympanum, eustachian tube, supratubary recess, anterior and posterior epitympanum, facial recess, oval and round window niche, and hypotympanum was performed. In the mastoid region, the aditus, antrum, and tip were also examined.
5. Endoscopic phase: Microscopic removal of any visible disease was conducted. It followed a routine of endoscopic revision of the middle ear and mastoid to identify possible foci of residual disease and improve the aeration routes. Mesotympanum, ossicular chain, and mastoid were visualized with a 0-degree endoscope. Protympanum, eustachian tube, supratubary recess, and tensor fold were inspected with a 30-degree endoscope angled forward. When the endoscope is motioned up, the tegmen, epitympanum, and aditum can be observed. A 30-degree pivot of the endoscope aims to visualize the facial recess, tympanic sinus, and oval and round windows.

6. Tympanoplasty with temporal fascia and/or cartilage

7. Suturing

The collected data were analyzed in a database using the SPSS SAS software (SAS Institute, Cary, NC, USA), studio version. The results are presented through descriptive measures, prevalence, mean, and standard deviation. The chisquared test was used to analyze data, and the results were considered statistically significant if $p<0.05$. This research was approved and registered (15-0608) by the ethics committee of the research group and graduate of Hospital de Clínicas de Porto Alegre.

\section{Results}

The mean age of the patients was $34.9+/-20.4$ years, and 22 patients were male.

Of the 32 cases, 17 (53.12\%) had residual cholesteatoma in the endoscopic phase. Minimal disease was found, usually fragments of the cholesteatoma matrix ( - Fig. 1). Locations with covert diseases are represented in -Figure 2.

The distribution of covert disease varied according to the cholesteatoma growth patterns. Disease frequency is shown in -Figure 3.

The previous classification can be summarized into cholesteatomas with any involvement of the pars tensa (posterior mesotympanic, two routes, or undetermined) or only pars flaccida involvement (posterior epitympanic). The prevalences of covert disease found were $43.75 \%$ for pars flaccida and $62.50 \%$ for pars tensa.

\section{Discussion}

Cholesteatoma surgery has improved significantly over the decades. These advances were possible due to the enhanced visualization of the minute structures of the middle ear. Microscopes with powerful lenses and light have made surgeries increasingly safe and effective. Microsurgery of the middle ear has become the gold standard for the treatment of chronic otitis media.

There are different operative techniques for the treatment of cholesteatoma. Complete removal of the disease is possible with a transcanal tympanotomy initially when cholesteatoma is restricted to the middle ear. However, most patients require mastoidectomy for complete pathology 


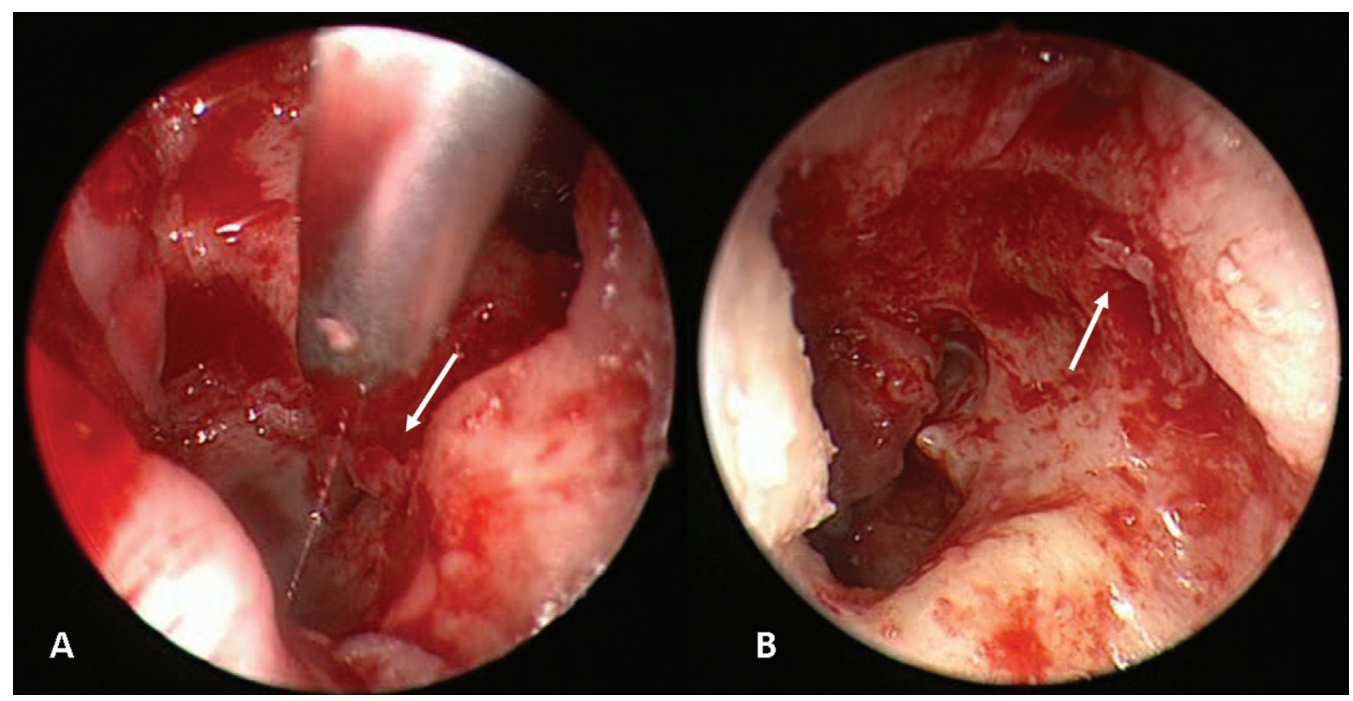

Fig. 1 Endoscopic view: residual cholesteatoma (arrows) after microscopic phase. A, posterior recesses. B, tegmen timpani.

removal. The most commonly used variants are CWU tympanomastoidectomy and canal wall-down mastoidectomy.

The CWU technique has some advantages. Preservation of the posterior wall of the EAC maintains the anatomy of the middle ear. This makes ossicular chain reconstruction tech- niques more successful and promotes optimal hearing results. Another benefit is being able to get the ears wet without protection. Such benefits are highlighted for people who engage in water sports, and they improve the quality of life of all patients.

\section{Location of covert disease}

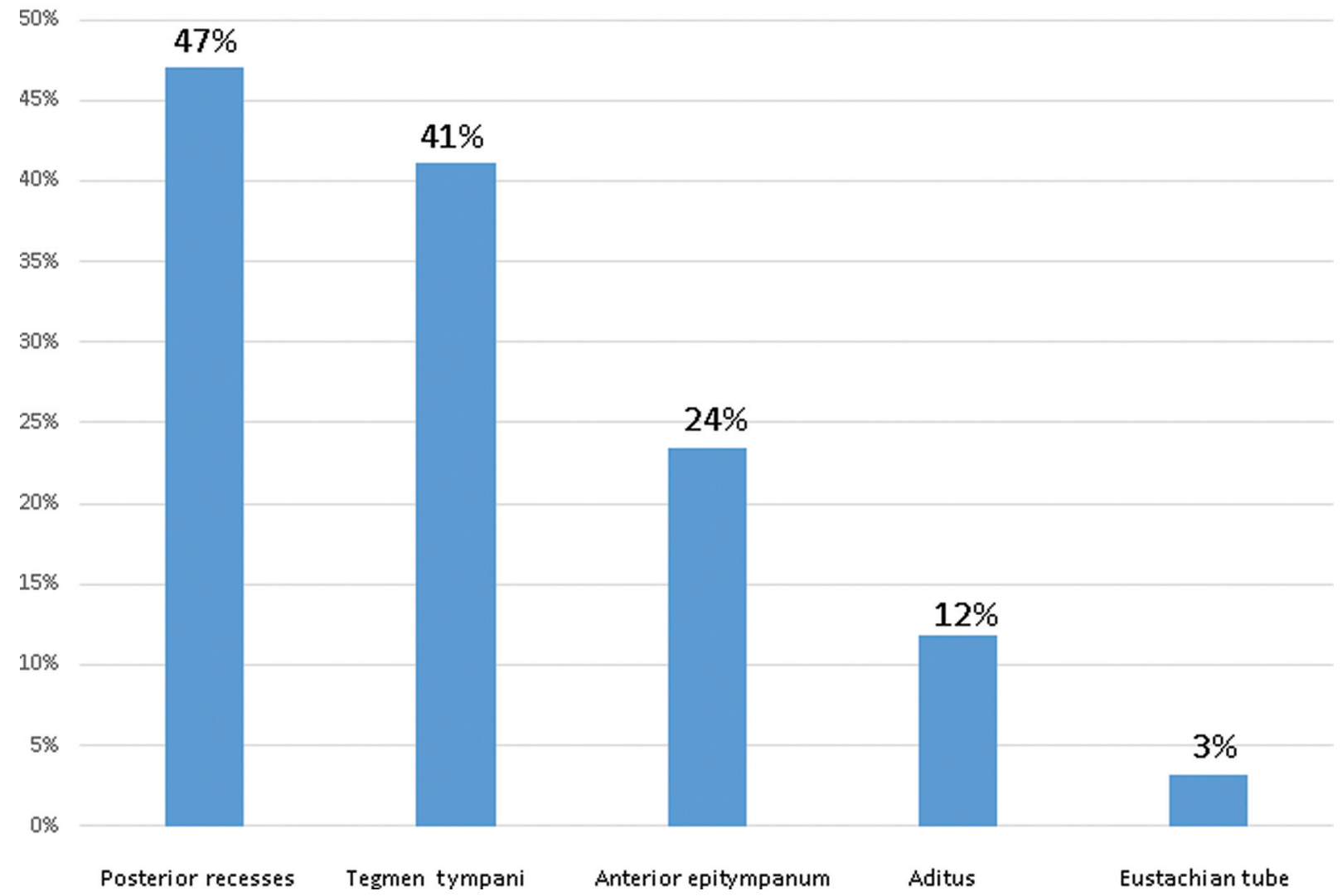

Fig. 2 Location of covert cholesteatoma identified with the use of endoscope. Y-axis shows the percentage of disease found at each site in relation to all locations. Comparison between the two most prevalent sites (posterior recesses and tegmen tympani) in relation to the other sites demonstrates a statistical difference $(p=0.0237)$. 


\begin{tabular}{ll}
\hline Growth Pattem & Prevalence of covert disease \\
\hline Posterior Mesotympanic & $5 / 9(55.5 \%)$ \\
Posterior epitympanic & $7 / 16(43.75 \%)$ \\
Two-route cholesteatoma & $3 / 5(60 \%)$ \\
Undetermined cholesteatoma & $2 / 2(100 \%)$ \\
\hline
\end{tabular}

Fig. 3 Prevalence of covert cholesteatoma in different growth patterns.

Nevertheless, the CWU technique also poses some pitfalls, which include high rates of recidivism. Preservation of the EAC wall hinders complete removal of the cholesteatoma in many cases. Sometimes, it is not possible to visualize some recesses of the middle ear, such as the facial recess and the tympanic sinus. The precarious nature of this curative procedure warrants the need for a "second-look" to unveil any recurrence. Currently, this second procedure can be replaced by diffusion-weighted imaging-magnetic resonance imaging (DWI-MRI) in selected cases. Despite the stringent postoperative care, cholesteatoma recurrence rates using this technique reach $30 \%$ in some series. 6,7

Endoscopic ear surgery is an alternative or complementary technique that reduces recidivism. Primarily, the use of endoscopy was to complement the microscope. When surgeons experience difficulty visualizing some specific areas, fiberoptics is used as this can be angled to determine, for example, total removal of the pathology. Moreover, endoscopic techniques already evolved, and surgeries could be performed without using a microscope. Currently, some surgeons advocate the use of totally endoscopic ear surgery of the middle ear. ${ }^{7}$

The advantages of the routine use of endoscopes in ear surgery include better visualization of all recesses of the middle ear and mastoid, certainty of the removal of the pathology, and the possibility of less invasive surgery since less bone drilling is required for surgical access.

Studies evaluating endoscopic surgery in chronic otitis media have progressively demonstrated its benefits. Existing research reveals good results from this operative technique and impels a change in the surgical practice of the middle ear. $^{8-10}$ In a historical cohort, James et al. compared the results of a group of patients operated with endoscopes and another with the conventional microscopic technique and demonstrated a small benefit in the second group. ${ }^{9}$ Arindam Das et al. ${ }^{11}$ conducted a randomized controlled trial and described a smaller bone drilling area and shorter surgical time in initial attic cholesteatomas.

Cholesteatoma is a recurrent disease. High recurrence rates and residual disease, regardless of the surgical technique employed, greatly affect the quality of life of patients. Studies show 15 to $17 \%$ new retraction pockets using the conventional closed tympanomastoidectomy technique and residual disease of 6 to $34 \%{ }^{6,7}$ The combined microscopic and endoscopic surgical technique was also evaluated and revealed a recurrence rate of $25 \%$ and residual disease in $6.5 \%$ of cases. ${ }^{12}$ The present study showed $53.12 \%$ transoperative covert disease after removal of the cholesteatoma with a microscope in the closed mastoidectomy technique. It is impossible to know if all these patients would develop a clinical relapse, mainly because the amount of disease found was very minimal. Most of the cases presented with thin fragments of adhered matrix. Perhaps, many of these patients never formed a new cholesteatoma, or they developed just a small pearl that allows easy removal in the second look. A prospective study with long-term recurrence assessment is warranted to answer this question.

It is also important to consider the commonly involved sites. In the present study, we found a significantly high prevalence of covert cholesteatoma in the posterior recesses and tegmen tympani. This is probably due to the difficulty in inspection using the microscope. Due to the anatomic curvature of the tegmen tympani, this allows concealment of the disease medially. The posterior recesses are certainly the most difficult location to be visualized and accessed using the closed technique. Their depth and angulations preclude the complete removal of the disease. The risk of recurrence is greatest in the tympanic sinus and facial recess, and cholesteatomas that settle in this location are more likely to relapse. The use of angled fiberoptics seems to overcome this problem. The higher prevalence of covert disease in pars tensa cholesteatomas is related to the formation pathway. This subtype grows through the posterior tympanic isthmus and inferior incudal space. It reaches the mastoid, invaginating medially to the ossicular chain. As a consequence, it precociously erodes the incudostapedial joint and reaches and fills the recesses of the facial and tympanic sinus before reaching the mastoid. Conversely, in the pars flaccida cholesteatoma, the Shrapnell membrane subsequently invaginates the Prussak space, goes through the upper incudal space laterally to the incus, crosses the aditus, and reaches the mastoid antrum. This cholesteatoma tends to reach the mesotympanum later on, through the von Tröltsch posterior pouch.

A potential bias in the present study is the presumption that the use of the endoscope would lead to less effective microscopic removal of the cholesteatoma. To overcome this error, we maintained a strict microscope inspection routine before proceeding with the endoscopic phase. Careful cleaning of the cholesteatoma was accomplished as evidenced by the negligible amounts of epithelium seen in all cases.

\section{Conclusion}

The use of fiberoptics in cholesteatoma surgery favors better visualization of covert disease. Cholesteatomas of the pars tensa presented more covert disease and was significantly more common in the posterior recesses and tegmen tympani. Prospective controlled studies are needed to determine the actual benefit of endoscopic ear surgeries in the long term. 
264 Endoscopic Assisted Canal Wall-up Tympanomastoidectomy Silva et al.

\section{Conflict of Interests}

The authors have no conflict of interests to declare.

\section{References}

1 Costa SS, Cruz OLM, Oliveira JAA. Otorhinolaryngology (Principles and Practice). 2nd ed. Porto Alegre: Artmed; 2006:309

2 Jackler RK, Santa Maria PL, Varsak YK, Nguyen A, Blevins NH. A new theory on the pathogenesis of acquired cholesteatoma: Mucosal traction. Laryngoscope 2015;125(Suppl 4):S1-S14

3 Rosito LS, Netto LF, Teixeira AR, da Costa SS. Classification of Cholesteatoma According to Growth Patterns. JAMA Otolaryngol Head Neck Surg 2016;142(02):168-172

4 Ghaffar S, Ikram M, Zia S, Raza A. Incorporating the endoscope into middle ear surgery. Ear Nose Throat J 2006;85(09):593-596

5 da Costa SS, Lavinsky M, Smith MM. Rock around the clock in the middle ear cleft. Laryngoscope 2002;112(06):1122-1125

6 James AL, Cushing S, Papsin BC. Residual Cholesteatoma After Endoscope-guided Surgery in Children. Otol Neurotol 2016;37 (02):196-201
7 Marchioni D, Soloperto D, Rubini A, et al. Endoscopic exclusive transcanal approach to the tympanic cavity cholesteatoma in pediatric patients: our experience. Int J Pediatr Otorhinolaryngol 2015;79(03):316-322

8 Lima TdeO, Araújo TF, Soares LC, Testa JR. The impact of endoscopy on the treatment of cholesteatomas. Rev Bras Otorrinolaringol (Engl Ed) 2013;79(04):505-511

9 Tarabichi M, Ayache S, Nogueira JF, Al Qahtani M, Pothier DD. Endoscopic management of chronic otitis media and tympanoplasty. Otolaryngol Clin North Am 2013;46(02):155-163

10 Badr-El-Dine M, James AL, Panetti G, Marchioni D, Presutti L, Nogueira JF. Instrumentation and technologies in endoscopic ear surgery. Otolaryngol Clin North Am 2013;46(02):211-225

11 Das A, Mitra S, Ghosh D, Sengupta A. Endoscopic Versus Microscopic Management of Attic Cholesteatoma: A Randomized Controlled Trial. Laryngoscope 2019;00:1-6

12 Neudert M, Lailach S, Lasurashvili N, Kemper M, Beleites T, Zahnert T. Cholesteatoma recidivism: comparison of three different surgical techniques. Otol Neurotol 2014;35(10): $1801-1808$ 